\title{
An investigation into the expression and mechanism of action of urotensin II in chronic pressure-overloaded rat hearts
}

\author{
WENYUAN LIU ${ }^{1}$, QINGHUA HAN ${ }^{1}$, QINGHUA LIU ${ }^{2}$, GANG LIANG $^{3}$, JIN WANG $^{4}$ and CHENGFANG LIU ${ }^{2}$ \\ ${ }^{1}$ Department of Cardiology, The First Hospital of Shanxi Medical University; \\ ${ }^{2}$ Teaching and Research Department of Pathophysiology, Shanxi Medical University; ${ }^{3}$ Department of Pathology, \\ The First Hospital of Shanxi Medical University; ${ }^{4}$ Teaching and Research Department of Physiology, \\ Shanxi Medical University, Taiyuan, Shanxi 030001, P.R. China
}

Received October 15, 2014; Accepted July 17, 2015

DOI: $10.3892 / \mathrm{mmr} .2015 .4244$

\begin{abstract}
The present study aimed to investigate the role and mode of action of urotensin II (U II) in the occurrence and progression of cardiac fibrosis in a pressure-overload rat model. Coarctation of the abdominal aorta was used to establish an animal model, and postoperative echocardiography, hemodynamic detection, hematoxylin and eosin staining, Masson staining and immunohistochemistry were performed to assess the changes in cardiac function and pathology. The expression levels of U II, G-protein-coupled receptor 14 and collagen $(\mathrm{Col}) \mathrm{I}$ and $\mathrm{Col}$ III in the myocardial tissues were also analyzed. Neonatal rat fibroblasts were isolated, cultured and subsequently, generations 3-5 were randomly divided into different groups for the detection of Col I and Col III levels by enzyme-linked immunosorbent assay and western blotting. The protein expression levels were markedly increased in the model group, and this increase correlated with an increase in myocardial fibrosis. In cultured neonatal rat fibroblast cells, $10^{-8} \mathrm{~mol} / 1 \mathrm{U}$ II significantly stimulated the synthesis of Col I and Col III $(\mathrm{P}<0.01)$ compared with the control group. Compared with the U II group, the administration of KT5720 (1 mol/l) or SB-611812 (1 mol/l) significantly reduced the synthesis and expression levels of $\mathrm{Col}$ I and Col III $(\mathrm{P}<0.05)$. U II may exert an important role in the process of myocardial fibrosis in chronic pressure-overload rats, and the cyclic adenosine monophosphate-protein kinase A signaling pathway may be partly involved in this process.
\end{abstract}

Correspondence to: Dr Qinghua Han, Department of Cardiology, The First Hospital of Shanxi Medical University, 85 Jiefangnan Road, Taiyuan, Shanxi 030001, P.R. China

E-mail: qinghuahandoc@126.com

Key words: urotensin II, coarctation of abdominal aorta, myocardial tissue, fibrosis, protein kinase

\section{Introduction}

Urotensin II (U II) is an effective vasoconstrictor present in different types of mammals, including humans, which has been demonstrated to be associated with a variety of cardiovascular diseases in previous years (1). U II is the most powerful vasoconstrictor known in the human body (2). The isolated human G-protein-coupled receptor 14 (UT) is the specific receptor for U II (2), and it is predominantly expressed in the brain, heart, kidney, adrenal gland and placenta $(3,4)$. The binding of U II to UT results in a range of biological effects $(4,5)$, specifically various cardiovascular effects, including promoting the growth of endothelial cells and vascular smooth muscle cells, cardiac fibrocyte proliferation, the constriction of vessels and lowering of the heart rate.

Previous studies demonstrated that in the aortic coarctation-induced pressure-overload rat model, plasma U II levels were markedly increased, and this was involved in the pressure overload-induced myocardial remodeling process (6). In addition, U II not only directly contributed to cardiac hypertrophy (7), but also promoted myocardial fibrosis by stimulating the proliferation of cardiac fibroblasts (8) and collagen synthesis (9). These results suggested that, in addition to affecting hemodynamics, U II may also be involved in myocardial fibrosis by increasing collagen synthesis (10). The administration of the UT antagonist, SB-611812, markedly improves the cardiac function of the experimental animals and markedly reduces myocardial remodeling (11). However, whether or not U II is involved in the occurrence and progression of myocardial fibrosis in the pressure-overload rat model, and the potential mechanism of action, remains to be elucidated. The present study aimed to investigate the association between myocardial fibrosis and systematic changes in the expression levels of U II and UT in a chronic pressure-overload rat model induced by coarctation of the abdominal aorta (CAA). The effect of changes in the expression levels of collagen (Col) I and Col III was also investigated. Simultaneously, a primary neonatal rat cardiac fibroblast cultivation model was established in order to investigate the effect of U II receptor antagonists and protein kinase A (PKA)-specific inhibitors on myocardial fibrosis. The effect of systematic changes in the expression levels of 
U II, UT, Col I and Col III was also investigated to assess the role of U II in myocardial fibrosis, and its association with the cyclic adenosine monophosphate (cAMP)-PKA signal transduction pathway in the chronic pressure-overload rat model.

\section{Materials and methods}

Materials. U II (rat) and KT-5720 were purchased from Tocris Bioscience (Bristol, UK); goat anti-rat U II polyclonal antibody (cat no. sc-21098) was purchased from Santa Cruz Biotechnology, Inc. (Santa Cruz, CA, USA); rabbit anti-rat anti-Col I (cat no. ab34710) and III (cat no. ab7778) polyclonal antibodies were purchased from Abcam (Cambridge, UK); goat anti-rabbit immunoglobulin G (IgG) PV-6000 working fluid was purchased from Xingsheng Biotech Co., Ltd. (Nanjing, China); Dulbecco's modified Eagle's medium, fetal bovine serum, collagenase and trypsin were purchased from Invitrogen Life Technologies (Carlsbad, CA, USA).

Animals. A total of 45 Sprague-Dawley rats weighing 200-220 g were supplied by the Experimental Animal Center of Shanxi Medical University (Shanxi, China) and were fed a regular diet. Animals were kept at $20 \pm 2^{\circ} \mathrm{C}$ and a relative humidity of $40-55 \%$ with a 12-h light/dark cycle. The experimental animal license number was SYXK:2006-0015. The animal experiments were performed according to the guidelines on experimental animal care and use, issued by the American National Institutes of Health (NIH Publication, 8th Edition, 2011) (12). The present study was performed strictly in accordance with the recommendations in the Guide for the Care and Use of Laboratory Animals of the National Institutes of Health. The animal use instructions were reviewed and approved by the Institutional Animal Care and Use Committee of Shanxi Medical University.

Establishment of the CAA model. Following skin preparation, the rats were anesthetized by intraperitoneal injection of $10 \%$ chloral hydrate $(3 \mathrm{ml} / \mathrm{kg}$; Chengdu Kelong Chemical Co. Ltd., Chengdu, China). An incision was made in the middle of sub-xiphisternal abdomen, and the abdominal cavity was opened layer by layer. The vagina vasorum of the abdominal aorta was stripped at the top of the left and right renal arteries, and the abdominal aorta was isolated. A tip-blunt no. 7 needle (Northwest Medical Devices Co., Ltd., Chengdu, China) was placed inside the aorta along the direction of the blood vessel, and a no. 4 surgical thread was used to ligate the abdominal aorta around the needle. The needle was slowly withdrawn to achieve partial stenosis of the abdominal aorta and the abdomen was subsequently closed layer by layer. In the sham group of animals, the surgical thread was only wound around the abdominal aorta following the opening of the abdomen; it was not used to ligate the abdominal aorta (13). On postoperative day 3, 100,000 units of penicillin (North China Pharmaceutical Co., Ltd., Shijiazhuang, China) were intraperitoneally injected to prevent infection. All animals were placed in the feeding room at a temperature of $24 \pm 3^{\circ} \mathrm{C}$ with artificial lighting (light and dark cycles of $12 \mathrm{~h}$ each). The animals were fed a standard rat diet and had free access to water. Group I consisted of control, sham-operated animals $(n=15)$; Group II consisted of 4 week post-surgery CAA rats $(n=10)$; Group III consisted of 8 week post-surgery CAA rats ( $\mathrm{n}=10)$; Group IV consisted of 12 week post-surgery CAA rats $(n=10)$.

Throughout the course of the experiment, all 15 sham-operated rats survived and the survival rate was $100 \%$. In the CAA group animals, 9 rats were alive 4 weeks following surgery (mortality rate 10\%), at 8 weeks post-surgery, 8 rats survived (mortality rate of 20\%) and at 12 weeks post-surgery, 6 rats survived (mortality rate of $40 \%$ ).

Echocardiographic examination. A General Electric Vivid 7 echocardiographic machine (GE Healthcare, Pittsburgh, PA, USA) was used to perform the echocardiography 4, 8 and 12 weeks following surgery. The rats were anesthetized using $10 \%$ chloral hydrate $(3 \mathrm{ml} / \mathrm{kg})$, their chest hair was removed and echocardiography was subsequently performed using a $10 \mathrm{~S}$ probe (the probe frequency was $11.0 \mathrm{MHz}$ ). Motion-mode ultrasound was used to record the curves of the left ventricle, the interventricular septum and the left ventricular (LV) posterior wall, using the parasternal long axis view. The end-diastolic interventricular septal thickness (IVSTd), end-diastolic LV posterior wall thickness (LVPWTd), end-diastolic LV diameter (LVDd, all measured in $\mathrm{mm}$ ) and LV ejection fraction (EF, recorded as a percentage), were measured. Each parameter was measured three times and the average was calculated.

Detection of the hemodynamic response. Following the induction of anesthesia, the rats were fixed on the operating table. A longitudinal incision $(1.5-2 \mathrm{~cm})$ was made in the neck skin and the right common carotid artery was separated. The common carotid artery at the distal end of heart was ligated and the proximal end was gripped with an artery clamp. A small opening was made in the common carotid artery and a $1 \mathrm{~mm}$ polyethylene catheter (American Health \& Medical Supply International Corp, Scarsdale, NY, USA), pre-filled with heparin saline, was inserted. A RM6240B-type biological-function experimental system (Chengdu Instrument Company, Chengdu, China) was used to record the LV systolic pressure (LVSP), the LV end-diastolic pressure (LVEDP) and the maximum increases and decreases in the LV pressures $( \pm \mathrm{dP} / \mathrm{dtmax})$.

Myocardial histological examination. Following the completion of the observation period (12 weeks), the rats were weighed and anesthetized, and a sample of $\sim 3 \mathrm{ml}$ abdominal aorta blood was obtained. The blood sample was subsequently centrifuged at $574 \mathrm{xg}$ for $15 \mathrm{~min}$ to separate the serum. The serum was subsequently cryopreserved at $-20^{\circ} \mathrm{C}$ for future experiments. The heart was rapidly removed and lavaged with pre-chilled saline. The surrounding tissues were dissected and the liquid on the surface, and inside the cardiac chambers was removed using filter paper (Sangon Biotech, Shanghai, China). The partial LV free-wall was subsequently cut and placed into $4 \%$ paraformaldehyde fixative prior to examination under a light microscope (CME; Leica Microsystems, Wetzlar, Germany). The myocardial tissue was paraffin-embedded prior to morphological examination of the myocardial cells, including hematoxylin and eosin (HE), and Masson staining.

Immunohistochemistry. Immunohistochemical staining was performed to determine the expression levels of U II, UT, $\mathrm{Col} \mathrm{I}$ and $\mathrm{Col}$ III in the cardiac tissues. Conventional paraffin 
Table I. Comparison of IVSTd, LVPWTd, LVDd and EF between the two groups.

\begin{tabular}{lcccccc}
\hline Group & N & Time (weeks) & IVSTd (mm) & LVPWTd (mm) & LVDd (mm) & EF (\%) \\
\hline Sham & 5 & 4 & $1.72 \pm 0.14$ & $1.74 \pm 0.12$ & $4.81 \pm 0.25$ & $72.32 \pm 5.68$ \\
& 5 & 8 & $1.85 \pm 0.11$ & $1.75 \pm 0.45$ & $4.85 \pm 0.16$ & $73.12 \pm 5.11$ \\
& 5 & 12 & $1.75 \pm 0.12$ & $1.76 \pm 0.13$ & $4.86 \pm 0.13$ & $74.15 \pm 5.16$ \\
Model & 6 & 4 & $2.34 \pm 0.11^{\mathrm{a}}$ & $1.84 \pm 0.25$ & $4.91 \pm 0.48$ & $70.70 \pm 5.94$ \\
& 6 & 8 & $2.40 \pm 0.03^{\mathrm{a}}$ & $2.52 \pm 0.24^{\mathrm{a}}$ & $6.83 \pm 0.28^{\mathrm{a}}$ & $63.15 \pm 5.23^{\mathrm{a}}$ \\
& 6 & 12 & $1.72 \pm 0.17^{\mathrm{b}}$ & $2.87 \pm 0.35^{\mathrm{b}}$ & $7.05 \pm 0.14^{\mathrm{b}}$ & $45.16 \pm 4.21^{\mathrm{b}}$ \\
\hline
\end{tabular}

Values are expressed as the mean \pm standard deviation. ${ }^{a} \mathrm{P}<0.05$ and ${ }^{\mathrm{b}} \mathrm{P}<0.01$, compared with the sham group. EF, ejection fraction; $\mathrm{LV}$, left ventricular; IVSTd, end-diastolic interventricular septal thickness; LVDd, end-diastolic left ventricular diameter; LVPWTd, end-diastolic left ventricular posterior wall thickness; $\mathrm{N}$, number.

sections were cut $2.0 \mu \mathrm{m}$ thick and washed with phosphate buffered saline (PBS) three times for $5 \mathrm{~min}$. Antigen retrieval was performed for $2 \mathrm{~min}$ in high-pressure hot citrate solution (Zhongshan Golden Bridge Biotechnology Co., Ltd., Beijing, China). The tissue sections were subsequently cooled to room temperature and washed three times for 2 min with PBS. The primary antibodies, anti-U II (1:150), anti-UT (1:200), anti-Col I (1:150) and anti-Col III (1:150) were applied dropwise, and incubated for $1 \mathrm{~h}$ at $37^{\circ} \mathrm{C}$. The tissue sections were cooled to room temperature for $60 \mathrm{~min}$ prior to washing three times for 2 min with PBS. The secondary antibody (goat anti-rabbit IgG) PV-6000 working solution (Zhongshan Golden Bridge Biotechnology Co., Ltd.) was subsequently added dropwise, and the tissue sections were incubated at $37^{\circ} \mathrm{C}$ for $20 \mathrm{~min}$, prior to washing three times for $2 \mathrm{~min}$ with PBS. The proteins were detected using diaminobenzidine staining, and were subsequently counterstained with hematoxylin prior to dehydration and mounting with neutral gum. The Aperio scanscope scanning system (Aperio, Buffalo Grove, IL, USA) was used for digital pathological scanning.

Determination of the cAMP content. The double-antibody sandwich ABC-ELISA kit (Senxiong Biotechnology Co., Ltd., Shanghai, China) was used to measure the plasma cAMP levels in the CAA model rats at 4,8 and 12 weeks, according to the manufacturer's instructions.

Cultivation of neonatal rat fibroblasts. A thoracotomy was performed on 1 to 3-day-old Sprague Dawley rats under aseptic conditions to obtain the hearts. Following digestion with collagenase $(0.04 \%)$ and trypsin $(0.08 \%)$ (Invitrogen Life Technologies), $10 \%$ serum-containing medium was added to form a cell suspension. In accordance with the different wall-adherence durations of fibroblasts and cardiomyocytes, differential adhesion was performed for $1.5 \mathrm{~h}$ in order to obtain the cardiac fibroblasts. The passaged fibroblasts were subsequently confirmed using an anti-vimentin monoclonal antibody (1:100; cat no. ab8978; Abcam, Cambridge, UK) and immunocytochemistry; the purity was $95 \%$.

Grouping. Generations 3-5 of the cells were used in the present study. The cells were grouped as follows: i) Control group with no stimulation; ii) U II group, where the cells were stimulated with $10^{-8} \mathrm{~mol} / 1 \mathrm{U} \mathrm{II}$; iii) U II+KT-5720 group, where the cells were stimulated with $10^{-8} \mathrm{~mol} / 1 \mathrm{U}$ II + $1 \mathrm{~mol} / 1 \mathrm{KT}-5720$; and iv) U II+SB-611812 group, where the cells were stimulated with $10^{-8} \mathrm{~mol} / 1 \mathrm{U} \mathrm{II}+1 \mathrm{~mol} / 1 \mathrm{SB}-6118$ 12. The changes in corresponding indexes of each group were observed at the specified time points.

The protein concentrations of Col I and Col III in the cell supernatants were determined using an ELISA [Rat Collagen Type I,III (Col I,III) ELISA kit; Tong Wei Biological Technology Co., Ltd., Shanghai, China] according to the manufacturer's instructions. The cells were seeded into 48-well plates using the above-mentioned method and were incubated with serum-free medium for $24 \mathrm{~h}$ prior to the application of the various stimuli for $48 \mathrm{~h}$. The supernatant was subsequently collected and the protein concentrations of Col I and Col III were determined by ELISA. The ELISA procedure was performed, according to the manufacturer's instructions, and each experiment was performed three times.

Western blotting. Following stimulation, the cell lysates were prepared using radioimmunoprecipitation assay buffer (Beyotime Institute of Biotechnology, Shanghai, China). The samples were mixed with $5 \mathrm{X}$ sample buffer, boiled for $5 \mathrm{~min}$ and centrifuged $\left(13,225 \mathrm{xg}, 15 \mathrm{~min}, 4^{\circ} \mathrm{C}\right)$. The protein concentration in the supernatant was determined using a bicinchoninic acid (BCA) protein quantitative kit (Wuhan Boster Biological Technology Co., Ltd.) according to the manufacturer's instructions. 10\% SDS-PAGE (Sangon Biotech) was performed using $50 \mu \mathrm{g}$ total protein from the cell lysates. Following electrophoresis, the proteins were transferred onto nitrocellulose membranes (Wuhan Boster Biological Technology, Co., Ltd.) and blocked with bovine serum albumin (Wuhan Boster Biological Technology Co., Ltd., Hubei, China) for $1 \mathrm{~h}$ at room temperature. The membranes were subsequently incubated with primary antibodies against Col I (1:5,000) and Col III (1:7,500) overnight at $4^{\circ} \mathrm{C}$. Following the washing of the membranes, the secondary antibodies were applied and the membranes were incubated for $1 \mathrm{~h}$, followed by enhanced chemiluminescence (ECL) detection using an ECL kit (Applygen Technologies Inc, Beijing, China). Band Scan Imaging software (Glyko, Novato, CA, USA) was used to analyze the intensity of the bands. 

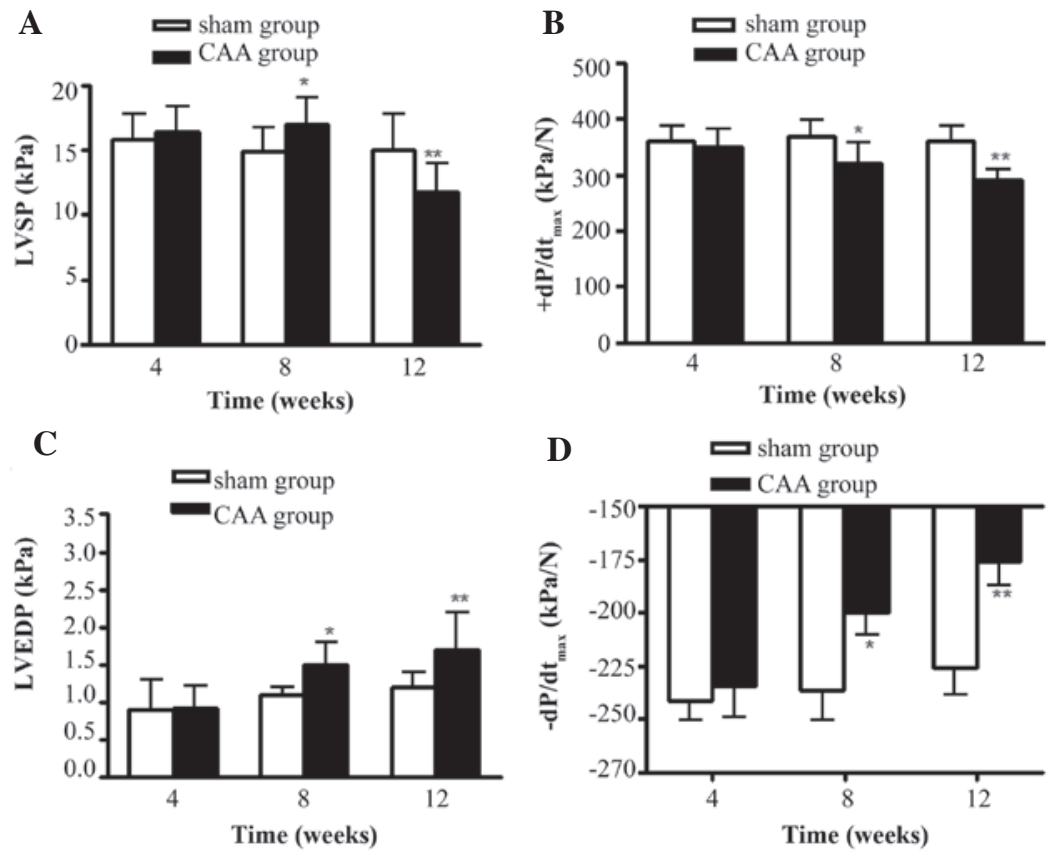

Figure 1. Changes in the hemodynamic parameters of each group. The data are shown for (A) LVSP, (B) $+d P / d t m a x,(C)$ LVEDP and (D) -dP/dtmax. ${ }^{*}<0.05$ and ${ }^{* *} \mathrm{P}<0.01$, compared with the control (sham) group $(\mathrm{n}=6)$. LV, left ventricular; LVSP , LV systolic pressure; LVEDP, LV end-diastolic pressure; $+\mathrm{dP} / \mathrm{dtmax}$, the maximum increase in the $\mathrm{LV}$ pressure; - $\mathrm{dP} / \mathrm{dtmax}$, the maximum decrease in the $\mathrm{LV}$ pressure.

Statistical analysis. SPSS 13.0 software (SPSS, Inc., Chicago, IL, USA) was used for statistical analysis. The quantitative data are expressed as the mean \pm standard deviation. $\mathrm{P}<0.05$ was considered to indicate a statistically significant difference.

\section{Results}

Echocardiography results. As shown in Table 1, at 4 weeks following surgery, the IVSTd of the model group had increased to a greater extent compared with the sham group, and the LVPWTd exhibited a non-significant thickening trend $(\mathrm{P}>0.05)$. The LVDd and EF were not significantly altered. The IVSTd, LVPWTd and LVDd of the model group were significantly increased relative to the sham group 8 weeks following surgery $(\mathrm{P}<0.05)$, and the EF was decreased $(\mathrm{P}<0.05)$. This trend continued for 12 weeks following surgery: The IVSTd, LVPWTd and LVDd of the model group were significantly increased relative to the sham group $(\mathrm{P}<0.01)$, and the $\mathrm{EF}$ was decreased $(\mathrm{P}<0.01)$.

Changes in the hemodynamic parameters. As shown in Fig. 1, compared with the sham group, the levels of LVSP, $+\mathrm{dP} / \mathrm{dtmax},-\mathrm{dP} / \mathrm{dtmax}$ and LVEDP were not significantly altered in the model group 4 weeks following surgery. The $+\mathrm{dP} / \mathrm{dtmax}$ and $-\mathrm{dP} / \mathrm{dtmax}$ levels were significantly decreased at 8 weeks following surgery $(\mathrm{P}<0.05)$, whereas the LVEDP was significantly increased $(\mathrm{P}<0.05)$. These changes were more pronounced at 12 weeks following surgery $(\mathrm{P}<0.01)$. The LVSP level temporarily increased 8 weeks following surgery $(\mathrm{P}<0.05)$ and significantly decreased 12 weeks following surgery $(\mathrm{P}<0.01)$.

Morphological examination of the myocardial tissue biopsies. HE staining revealed that, compared with the sham group, the diameter of the myocardial cells in the model group was increased. The cells in the model group were arranged irregularly with disorganized muscle fibers; the muscular fibers were loose and exhibited edema, with increased gaps between them, and the fibrous connective tissues around the partial muscle bundles exhibited hyperplasia (Fig. 2). Masson staining highlighted the collagen fibers with a blue coloration, whereas the muscle fibers and cellulose were colored red. Compared with the sham group, the expression of collagen fibers in the model group cardiac tissues gradually increased, particularly by the week 12 (Fig. 2).

Immunohistochemical staining results. Fig. 3 demonstrated the expression of U II in the rat myocardial tissues. The darker areas indicated U II expression inside the myocardial interstitium. Compared with the sham group, U II expression in the model group gradually increased over time. The quantitative analysis revealed that the increased U II expression in the model group was time-dependent $(\mathrm{P}<0.05)$.

Fig. 4 demonstrated the expression of UT in the rat myocardial tissues; the darker areas of staining indicate UT expression in the cytoplasm of the myocardial cells. There was a low expression level of UT in the cytoplasm of the sham group myocardial cells. Compared with the sham group, the expression of UT in the model group gradually increased over time. The quantitative analysis revealed that the increased expression of UT in the model group was time-dependent $(\mathrm{P}<0.05)$.

Figs. 5 and 6 revealed the expression levels of Col I and Col III in the rat myocardial tissues; the darker areas of staining indicated the expression of Col I and Col III in the myocardial interstitial tissues. There was a small quantity of $\mathrm{Col} \mathrm{I}$ and $\mathrm{Col}$ III expression in the sham group. Compared with the sham group, the expression levels of Col I and Col III in the 

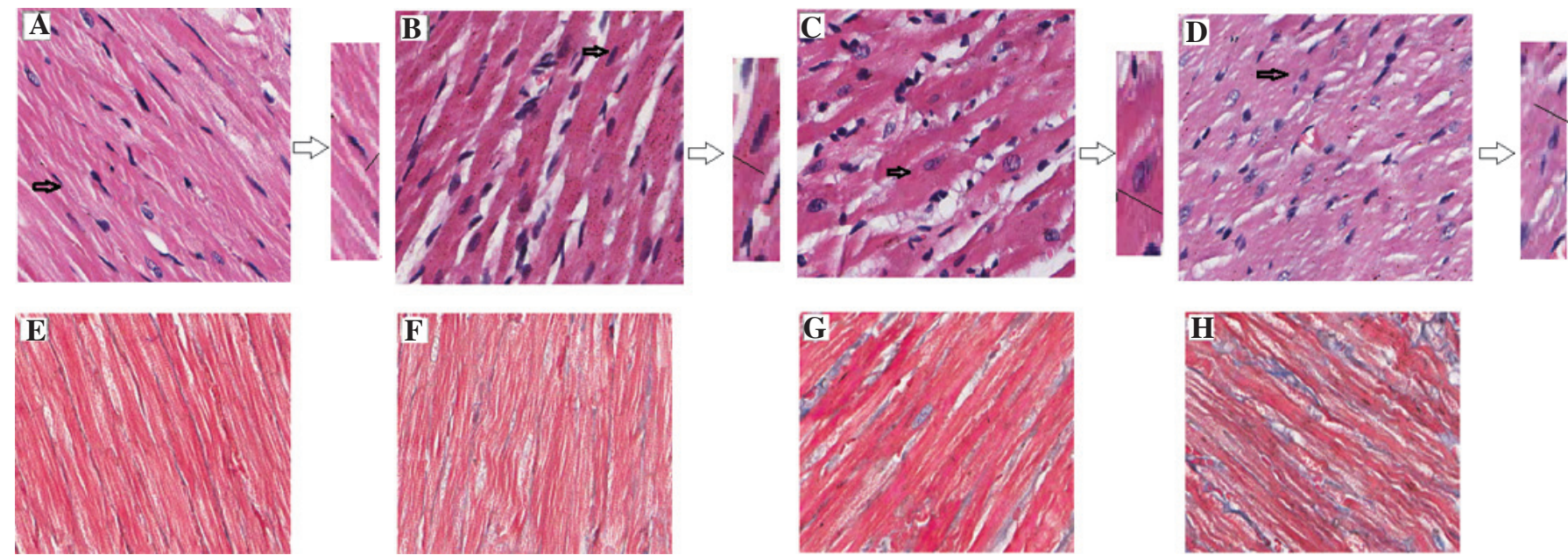

Figure 2. Photomicrographs of hematoxylin and eosin staining for the (A) sham group, (B) 4, (C) 8 and (D) 12 weeks following surgery model groups, and Masson staining for the (E) sham group, (F) 4 weeks, (G) 8 weeks, and (H) 12 weeks following surgery model groups. Magnification, x400. Arrows indicate a single typical cardiomyocyte (magnification, $\mathrm{x} 800$ ).

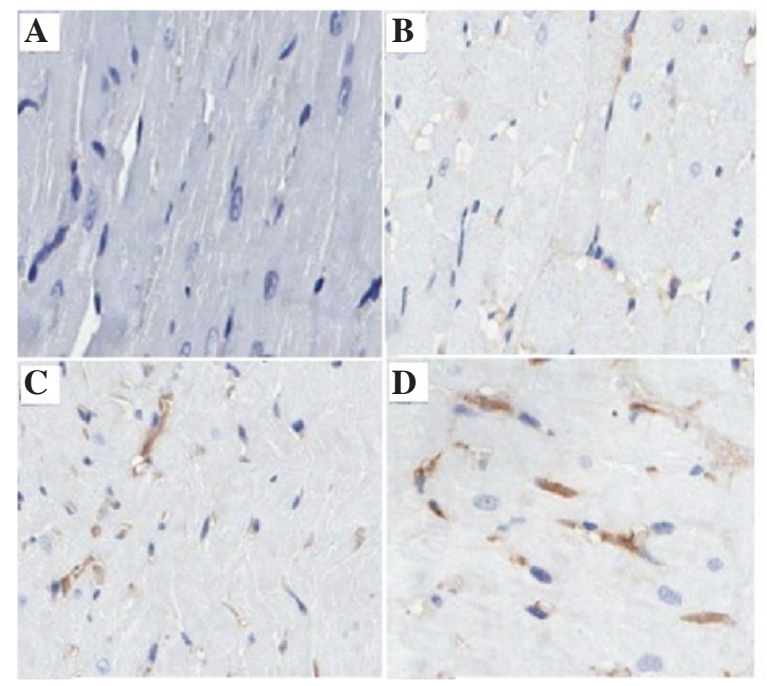

$\mathbf{E}$

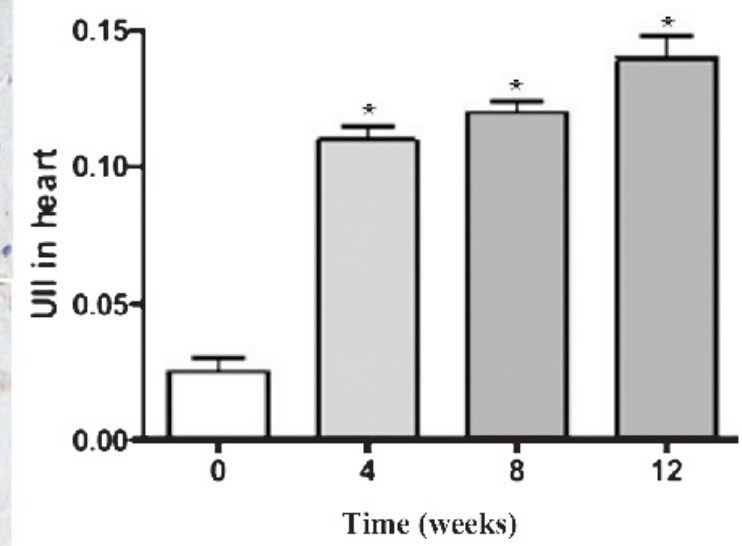

Figure 3. Changes in the expression of U II in the rat myocardial tissue. The darker areas indicate positive expression (magnification, $\mathrm{x} 400$; scale bar, $50 \mu \mathrm{m}$ ) The tissue in each panel represents the (A) sham group, (B) 4, (C) 8 and (D) 12 weeks following surgery model groups. (E) The bar chart illustrates the quantification of the expression of U II (mol/l). ${ }^{*} \mathrm{P}<0.05$, data compared with the pseudo-operation group. U II, urotensin II.
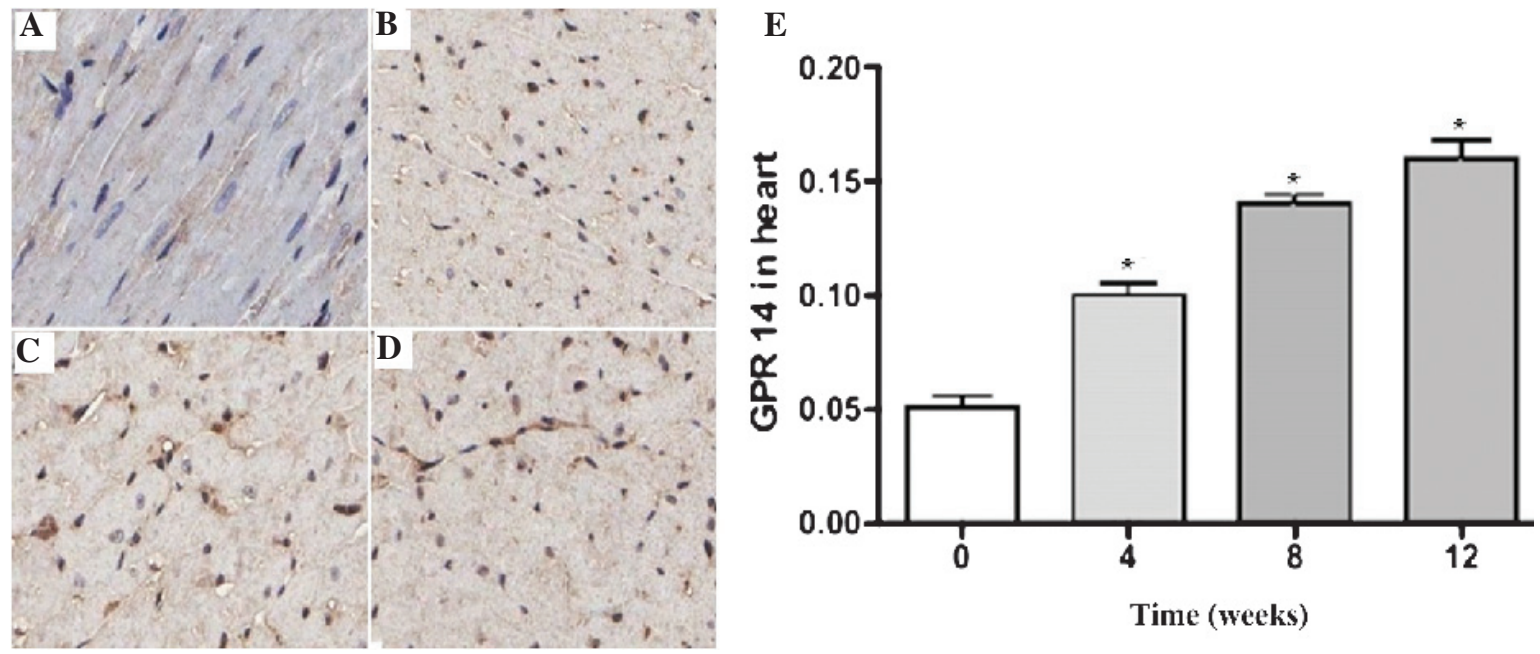

Figure 4. Changes in the expression of GPR14 in the rat myocardial tissues. The darker areas indicate the positive expression as before. The tissue shown in each panel represents the (A) sham group; (B) 4, (C) 8 and (D) 12 weeks following surgery model groups. (E) The bar chart illustrates the quantification of the expression of GPR14 (mol/l). " $\mathrm{P}<0.05$, data compared with the pseudo-operation group. GPR14, G-protein-coupled receptor 14. 


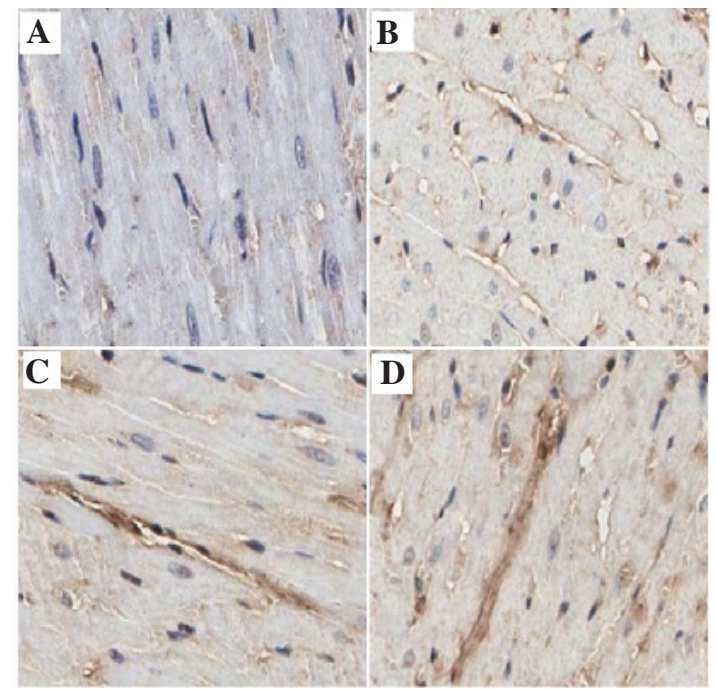

$\mathbf{E}$

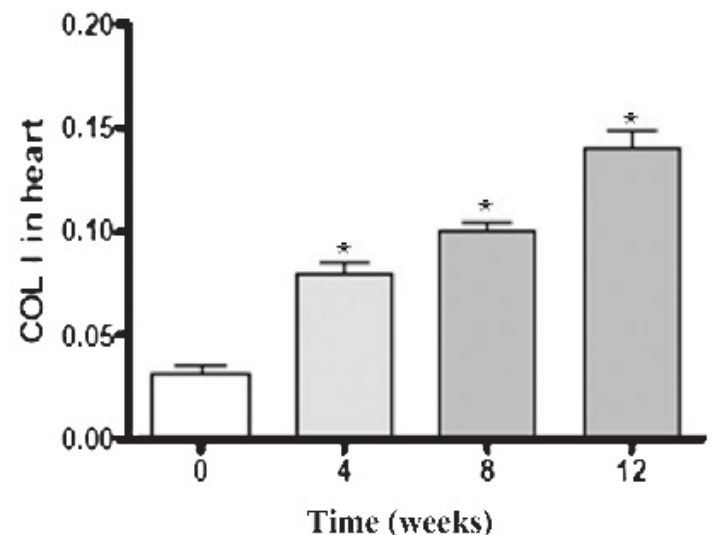

Figure 5. Changes in the expression of $\mathrm{Col} \mathrm{I}$ in the rat myocardial tissues. The darker areas indicate the positive expression as before. The tissue shown in each panel represents the (A) sham group, (B) 4, (C) 8 and (D) 12 weeks following surgery model groups. (E) The bar chart illustrates the quantification of the expression of $\mathrm{Col} \mathrm{I}(\mathrm{mol} / \mathrm{l}) .{ }^{*} \mathrm{P}<0.05$, data compared with the pseudo-operation group. Col I, collagen I.
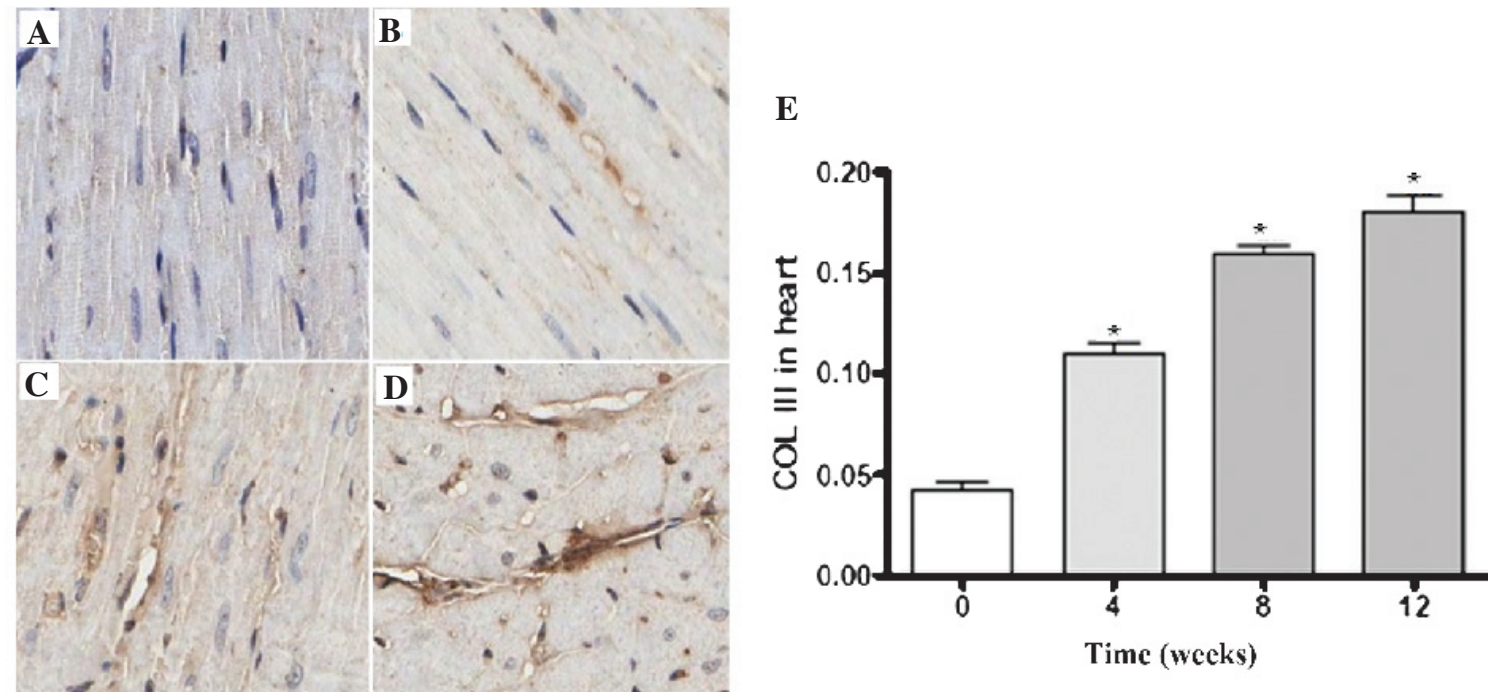

Figure 6. Changes in the expression of $\mathrm{Col}$ III in the rat myocardial tissues. The darker areas indicate the positive expression as before. The tissue shown in each panel represents the (A) sham group, (B) 4, (C) 8 and (D) 12 weeks following surgery model groups. (E) The bar chart illustrates the quantification of the expression of $\mathrm{Col}$ II (mol/l). $\mathrm{P}<0.05$, data compared with the pseudo-operation group. Col III, collagen III.

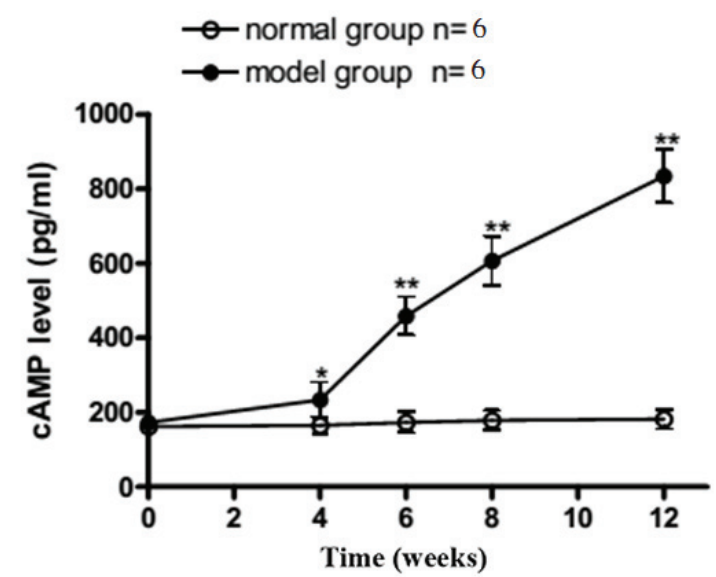

Figure 7. Changes in the plasma cAMP concentration of the model group measured at different time points. ${ }^{*} \mathrm{P}<0.05$ and ${ }^{* *} \mathrm{P}<0.01$, the model group compared with the control group $(n=6)$. cAMP, cyclic adenosine monophosphate. model group gradually increased over time. The quantitative analysis revealed that the increased expression of $\mathrm{Col} \mathrm{I}$ and $\mathrm{Col}$ III in the model group was time-dependent $(\mathrm{P}<0.05)$.

cAMP concentration determination using the ABC-ELISA method. As shown in Fig. 7, the plasma cAMP concentration in the model group was higher compared with the sham group at week $4(\mathrm{P}<0.05)$. At weeks 8 and 12 , the plasma cAMP concentrations exhibited a time-dependent increase, which was more significant compared with that observed in week $4(\mathrm{P}<0.01)$.

Determination of Col I and ColIII concentration using the ELISA method. As shown in Fig. 8, stimulation with $10^{-8} \mathrm{~mol} / \mathrm{l} \mathrm{U}$ II significantly stimulated the accumulation of Col I and Col III protein in the fibroblasts. This difference was statistically significant when compared with the control group $(\mathrm{P}<0.01)$. The 

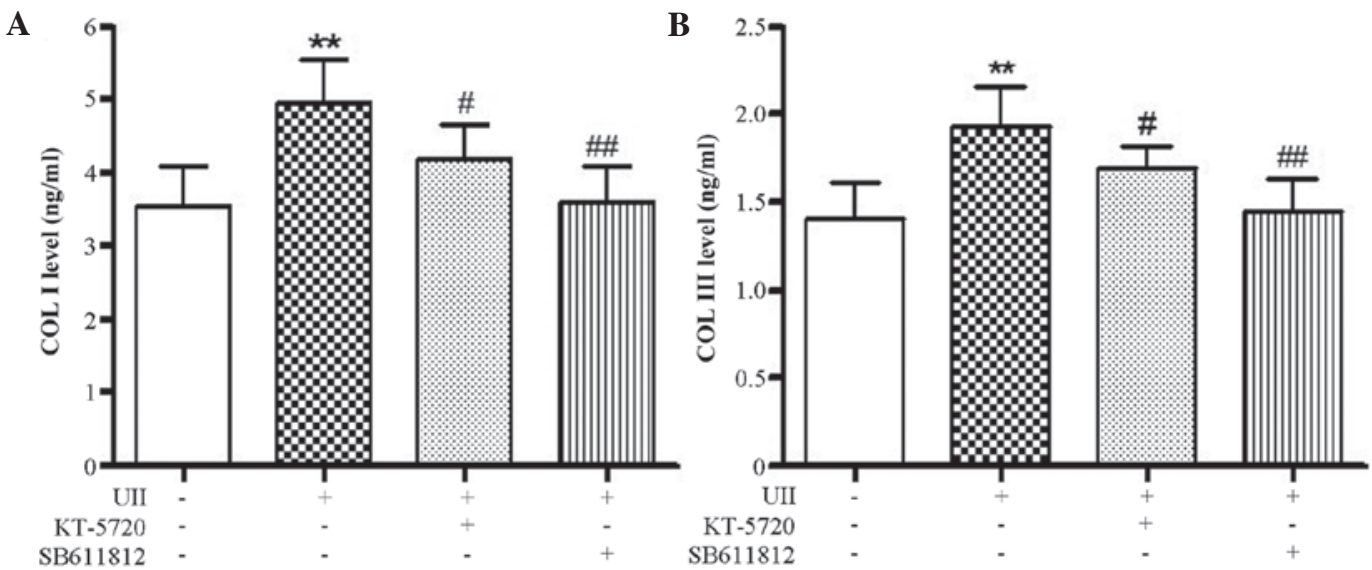

Figure 8. Quantity of (A) Col I and (B) Col III measured in the cellular supernatant of each group (the control group, or following treatment with the U II receptor antagonist, SB-611812, or the protein kinase A-specific inhibitor, KT-5720. ${ }^{* *} \mathrm{P}<0.01$, compared with the control group; ${ }^{\# P}<0.05$ and ${ }^{\# \#} \mathrm{P}<0.05$, compared with the U II group. Col, collagen; U II, urotensin II.
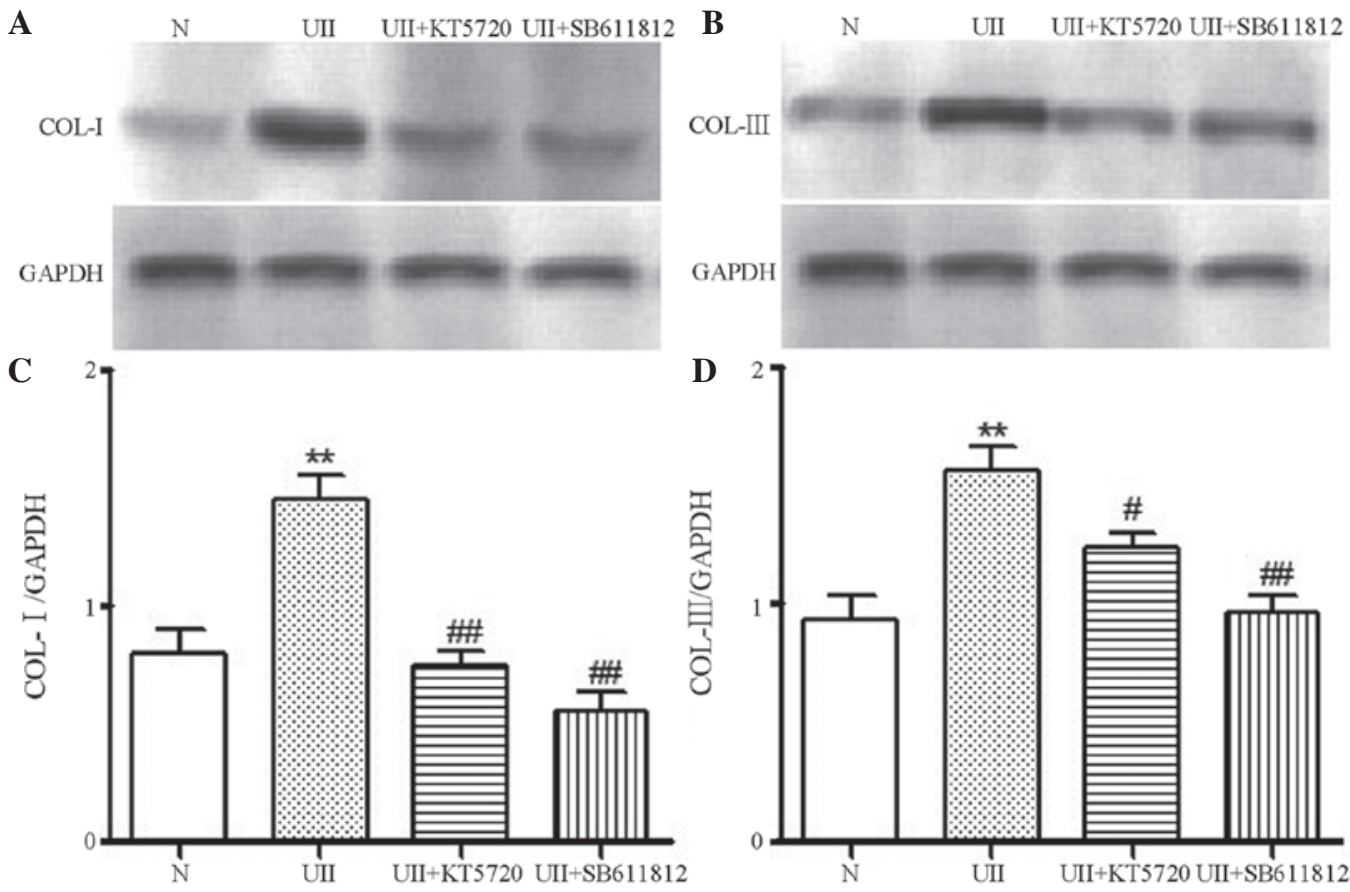

Figure 9. Expression of (A) Col I and (B) Col III in the fibroblasts of each group, when treated with U II alone or in combination with the U II receptor antagonist, SB-611812, or the protein kinase A-specific inhibitor, KT-5720. (C and D) ${ }^{*} \mathrm{P}<0.01$, compared with the control group; ${ }^{*} \mathrm{P}<0.05$ and ${ }^{\# \#} \mathrm{P}<0.05$, compared with the U II group. Col, collagen; U II, urotensin II.

administration of $10^{-8} \mathrm{~mol} / \mathrm{l} \mathrm{U}$ II and either the U II receptor antagonist, SB-611812, or the PKA-specific inhibitor, KT-5720, resulted in a significant decrease in Col I and Col III levels compared with the U II treatment group $(\mathrm{P}<0.05)$. These results indicated that the U II-stimulated synthesis of Col I and Col III in fibroblasts may be achieved through the PKA pathway.

Protein expression levels of Col I and Col III in fibroblasts. As shown in Fig. 9, there was a small quantity of Col I and Col III expression in the fibroblasts from the control group. Following stimulation with $10^{-8} \mathrm{~mol} / 1 \mathrm{U}$ II, the expression of $\mathrm{Col} \mathrm{I}$ and Col III increased significantly $(\mathrm{P}<0.01)$. The administration of $10^{-8} \mathrm{~mol} / \mathrm{l} \mathrm{U}$ II and either the U II receptor antagonist, SB-611812, or the PKA-specific inhibitor, KT5720, resulted in a significant decrease in Col I and Col III levels compared with the U II treatment group $(\mathrm{P}<0.05)$. These results indicated that the PKA pathway may be involved in the U II-stimulated synthesis of Col I and Col III in the fibroblasts.

\section{Discussion}

Heart failure is an important condition, which endangers human lives and chronic pressure-overload is an important cause of heart failure. $\mathrm{LV}$ pressure overload can induce cardiac hypertrophy, fibrosis and systolic dysfunction (14-16). As the duration of LV pressure overload increases, LV hypertrophy can gradually develop, eventually leading to heart failure $(14,15)$. CAA has been demonstrated to successfully replicate chronic 
pressure-overload heart failure and provides an appropriate experimental model (17).

U II was identified as a type of vasoactive peptide in mammals. Previous studies revealed that U II serum levels were markedly higher in patients with clinical heart failure $(18,19)$, and were also higher in the cardiac tissues of patients with end-stage heart failure (2). Similarly, animal experiments revealed that, in the coronary artery ligation-induced myocardial infarction heart failure rat model, the expression of ventricular U II and its receptor UT revealed a gradual time-dependent increase in concentration (7). The chronic perfusion of U II into rats can induce diastolic dysfunction and stimulate collagen synthesis (20). U II can also induce the mRNA expression levels of Col I and Col III in neonatal cardiac fibroblasts through the transforming growth factor $\beta 1$ signaling pathway $(21,22)$. These previous studies indicated that U II exerts an important role in heart failure and myocardial fibrosis. A study performed previously on the role and signal transduction mechanisms of U II have principally concentrated on blood vessel regulation (23). However, whether U II is involved in the development of chronic pressure in overload-induced rat myocardial fibrosis, and the possible mechanisms of this involvement, remain to be elucidated. In the present study, CAA was used to establish a rat model, and echocardiography and hemodynamic detection indicated that, with a prolonged modeling time, the systolic and diastolic functions of the model group rats decreased, confirming the use of the chronic pressure-overload heart failure model.

Furthermore, HE and Masson staining confirmed that the CAA-established chronic pressure-overload heart failure rat model exhibited clear myocardial fibrosis. Additionally, the degree of fibrosis gradually increased as the modeling time increased. Immunohistochemical staining revealed that the protein expression levels of U II, UT, Col I and Col III in the myocardial tissues of the model group markedly increased. As myocardial fibrosis progressed, the levels of these proteins exhibited a time-dependent increase, suggesting that U II and its receptor, UT, may be involved in the occurrence and development of myocardial fibrosis in the pressure-overload rats.

Contemporary studies have demonstrated that, in addition to its effects on hemodynamics, U II may be involved in myocardial fibrosis by increasing collagen synthesis (10). The UT antagonist, SB-611812, markedly reduced cardiac remodeling (11). The mechanism of U II-induced collagen synthesis may involve the PKC, mitogen-activated protein kinase or calcineurin (24) pathways, although the precise mode of U II action in myocardial cells remains to be fully elucidated (25-27). The cAMP-PKA pathway is a major pathway of cellular signal transduction, which regulates multiple protein activities, the expression of numerous genes and diverse cellular functions. The specific action of cAMP in cells is considered to be regulated via the activation of the cAMP-dependent PKA signaling pathway (28). Our previous study used the Langendorff rat isolated-heart model and observed that different concentrations of U II $\left(\mathrm{EC}_{50} 10^{-8} \mathrm{~mol} / \mathrm{l}\right)$ produced a dose-dependent inhibition of cardiac function; a more marked inhibitory effect was observed in the CAA-induced heart failure rat model (29). The PKA-specific inhibitor, KT5720, inhibits the U II-mediated heart function inhibition in normal and heart failure model rats, and at the cellular level, KT5720 was observed to inhibit the U II-stimulated inhibition of L-type $\mathrm{Ca}^{2+}$ currents in rat cardiomyocytes. Therefore, U II may inhibit cardiac L-type $\mathrm{Ca}^{2+}$ currents through the PKA pathway and this may account for the inhibitory effect of U II on cardiac functions (30). In order to further investigate the mechanism(s) of U II in myocardial fibrosis and the association with the cAMP-PKA pathway, an in vitro neonatal rat fibroblast experiment was performed. The administration of U II and the antagonists, KT5720 and SB-611812, indicated that $10^{-8} \mathrm{~mol} / 1 \mathrm{U}$ II significantly stimulated the synthesis of Col I and Col III in fibroblasts, and that KT5720 and SB-611812 significantly reduced the U II-stimulated synthesis and expression of Col I and Col III. These results indicated that KT5720 and SB-611812 significantly inhibited U II-induced Col synthesis in cardiac fibroblasts. Taken together with the in vivo experiments, which deomonstrated that plasma cAMP concentrations in the model group gradually increased with the severity of myocardial fibrosis, the results suggested that the cAMP-PKA signaling pathway may regulate U II-promoted collagen synthesis in cardiac fibroblasts, and therefore is involved in the process of pressure overload-induced myocardial fibrosis in rats.

In conclusion, in the CAA-induced chronic pressure-overload rat model, the extent of heart failure and myocardial fibrosis gradually increased with time. Similarly, the expression levels of U II, UT, Col I and Col III in myocardial tissues significantly increased with time, suggesting that U II may exert an important role in the myocardial fibrosis process in the pressure-overload rat model. The in vitro experiments revealed that the CAMP-PKA signaling pathway regulated the effects of U II on Col synthesis in cardiac fibroblasts, and that this effect was mediated by UT and antagonized by UT inhibition. Therefore a novel signaling pathway associating U II and myocardial fibrosis was putatively been identified, although further studies are required in animal models.

\section{Acknowledgements}

This study was supported by the Natural Science Foundation of Shanxi Province (no. 2012011036-1), the Shanxi Provincial Scientific Research Projects Foundation of Abroad-Studying Personnel (no. 2012-7), the Shanxi Provincial University Scientific Research Projects Foundation of Abroad-Studying and Returning Personnel (no. 2011-63), the Selected Scientific Research Projects Foundation of Abroad-Studying Personnel, Office of Human Resources, Shanxi Province (no. 2013-68), the Selected Scientific Research Projects Foundation of Abroad-Studying and Returning Personnel, the Shanxi Province (no. 2010-97), Technology Innovation Foundation of Shanxi Medical University (no. 2010-7) and the Shanxi Provincial Scientific Research Projects Foundation of Abroad-Studying and Returning Personnel (no. 2009-9).

\section{References}

1. Ross B, McKendy K and Giaid A: Role of urotensin II in health and disease. Am J Physiol Regul Integr Comp Physiol 298: R1156-R1172, 2010.

2. Ames RS, Sarau HM, Chambers JK, Willette RN, Aiyar NV, Romanic AM, Louden CS, Foley JJ, Sauermelch CF, Coatney RW, et al: Human urotensin-II is a potent vasoconstrictor and agonist for the orphan receptor GPR14. Nature 401: 282-286, 1999. 
3. Totsune K, Takahashi K, Arihara Z, Sone M, Satoh F, Ito S, Kimura Y, Sasano H and Murakami O: Role of urotensin II in patients on dialysis. Lancet 358: 810-811, 2001.

4. Matsushita M, Shichiri M, Imai T, Iwashina M, Tanaka H, Takasu $\mathrm{N}$ and Hirata Y: Co-expression of urotensin II and its receptor (GPR14) in human cardiovascular and renal tissues. J Hypertens 19: 2185-2190, 2001.

5. Ban Y, Watanabe T, Suguro T, Matsuyama TA, Iso Y, Sakai T, Sato R, Idei T, Nakano Y, Ota H, et al: Increased plasma urotensin-II and carotid atherosclerosis are associated with vascular dementia. J Atheroscler Thromb 16: 179-187, 2009.

6. Chen Z, Xu J, Ye Y, Li Y, Gong H, Zhang G, Wu J, Jia J, Liu M, Chen Y, et al: Urotensin II inhibited the proliferation of cardiac side population cells in mice during pressure overload by JNK-LRP6 signalling. J Cell Mol Med 18: 852-862, 2014.

7. Tzanidis A, Hannan RD, Thomas WG, Onan D, Autelitano DJ, See F, Kelly DJ, Gilbert RE and Krum H: Direct actions of urotensin II on the heart: Implications for cardiac fibrosis and hypertrophy. Circ Res 93: 246-253, 2003.

8. Shi L, Ding W, Li D, Wang Z, Jiang H, Zhang J and Tang C: Proliferation and anti-apoptotic effects of human urotensin II on human endothelial cells. Atherosclerosis 188: 260-264, 2006.

9. Zhang YG, Li J, Li YG and Wei RH: Urotensin II induces phenotypic differentiation, migration, and collagen synthesis of adventitial fibroblasts from rat aorta. J Hypertens 26: 1119-1126, 2008.

10. Zhao J, Ding W, Song N, Dong X, Di B, Peng F and Tang C: Urotensin II-induced collagen synthesis in cultured smooth muscle cells from rat aortic media and a possible involvement of transforming growth factor- $\beta 1 / \mathrm{Smad} 2 / 3$ signaling pathway. Regul Pept 182: 53-58, 2013

11. Bousette N, Pottinger J, Ramli W, Ohlstein EH, Dhanak D, Douglas SA and Giaid A: Urotensin-II receptor blockade with SB-611812 attenuates cardiac remodeling in experimental ischemic heart disease. Peptides 27: 2919-2926, 2006

12. National Research Council (US) Committee for the Update of the Guide for the Care and Use of Laboratory Animals: Guide for the Care and Use of Laboratory Animals. 8th edition. National Academies Press, Washington (DC), 2011.

13. Ma L, Liu J, Chu N, et al: The relationship between cardiac hypertrophy index and cardiac function in chronic heart failure rats. J Capit Med Univ (Chin) 31: 596-599, 2010.

14. Kehat I and Molkentin JD: Molecular pathways underlying cardiac remodeling during pathophysiological stimulation. Circulation 122: 2727-2735, 2010.

15. Berenji K, Drazner MH, Rothermel BA and Hill JA: Does load-induced ventricular hypertrophy progress to systolic heart failure? Am J Physiol Heart Circ Physiol 289: H8-H16, 2005.

16. Creemers EE and Pinto YM: Molecular mechanisms that control interstitial fibrosis in the pressure-overloaded heart. Cardiovasc Res 89: 265-272, 2011.

17. Bayer AL, Heidkamp MC, Patel N, Porter MJ, Engman SJ and Samarel AM: PYK2 expression and phosphorylation increases in pressure overload-induced left ventricular hypertrophy. Am J Physiol Heart Circ Physiol 283: H695-H706, 2002.
18. Lapp H, Boerrigter G, Costello-Boerrigter LC, Jaekel K, Scheffold T, Krakau I, Schramm M, Guelker H and Stasch JP: Elevated plasma human urotensin-II-1ike immunoreactivity in ischemic cardiomyopathy. Int J Cardiol 94: 93-97, 2004

19. Russell FD, Meyers D, Galbraith AJ, Bett N, Toth I, Kearns P and Molenaar P: Elevated plasma levels of human urotensin-II immunoreactivity in congestive heart failure. Am J Physiol Heart Circ Physiol 285: H1576-H1581, 2003.

20. Tran L, Kompa AR, Kemp W, Phrommintikul A, Wang BH and Krum H: Chronic urotensin-II infusion induces diastolic dysfunction and enhances collagen production in rats. Am J Physiol Heart Circ Physiol 298: H608-H613, 2010.

21. Dai HY, Kang WQ, Wang X, Yu XJ, Li ZH, Tang MX, Xu DL, Li CW, Zhang Y and Ge ZM: The involvement of transforming growth factor-beta1 secretion in urotensin II-induced collagen synthesis in neonatal cardiac fibroblasts. Regul Pept 140: 88-93, 2007.

22. Dai HY, He T, Li XL, Xu Wl and Ge ZM: Urotensin-2 promotes collagen synthesis via ERK1/2-dependent and ERK1/2-independent TGF- $\beta 1$ in neonatal cardiac fibroblasts. Cell Biol Int 35: 93-98, 2011.

23. Domínguez-Rodríguez A, Díaz I, Rodríguez-Moyano M, Calderón-Sánchez E, Rosado JA, Ordóñez A and Smani T: Urotensin-II signaling: Mechanism in rat coronary artery: Role of STIM1 and Orail-dependent store operated calcium influx in vasoconstriction. Arterioscler Thromb Vasc Biol 32: 1325-1332, 2012.

24. Song QG, Lü JZ and Yang JX: Effect of urotensin II on the proliferation of cardiac Myofibroblasts and its intracellular signaling mechanism in new-born SD rats. J Xi'an Jiaotong Univ 28: 157-160, 2007.

25. Kemp W, Kompa A, Phrommintikul A, Herath C, Zhiyuan J, Angus P, McLean C, Roberts S and Krum H: Urotensin II modulates hepatic fibrosis and portal hemodynamic alterations in rats. Am J Physiol Gastrointest Liver Physiol 297: G762-G767, 2009.

26. Rossowski WJ, Cheng BL, Taylor JE, Datta R and Coy DH: Human urotensin II-induced aorta ring contractions are mediated by protein kinase $\mathrm{C}$, tyrosine kinases and Rho-kinase: Inhibition by somatostatin receptor antagonists. Eur J Pharmacol 438: 159-170, 2002.

27. Zhang YG, Li YG, Liu BG, Wei RH, Wang DM, Tan XR, Bu DF, Pang YZ and Tang CS: Urotensin II accelerates cardiac fibrosis and hypertrophy of rats induced by isoproterenol. Acta Pharmacol Sin 28: 36-43, 2007.

28. Staudt Y, Mobini R, Fu M, Felix SB, Kühn JP and Staudt A: Beta1-adrenoceptor antibodies induce apoptosis in adult isolated cardiomyocytes. Eur J Pharmacol 466: 1-6, 2003.

29. Li CJ, Han QH: Effect of Urotensin II on cardiac function in heart failure rats and its mechanism of action. Chinese J Integr Med Cardio Cerebrovasc Dis 6: 38-40, 2008.

30. Han QH, Liu WY, Li CJ, Wang R and Shi HT: The effect of urotensin II on cardiac function of rats and its electrophysiological mechanism. Chinese J Integr Med Cardio Cerebrovasc Dis 9: 1479-1481, 2011. 